\title{
ENTROPÍA PROPORCIONAL DE DINÁMICAS CARDIACAS NORMALES Y CON ARRITMIA EN LA UNIDAD DE CUIDADOS INTENSIVOS
}

\author{
PROPORTIONAL ENTROPY OF NORMAL CARDIAC DYNAMIC AND WITH ARRHYTHMIA IN THE \\ INTENSIVE CARE UNIT
}

Javier O Rodríguez Velasquez', Signed E Prieto Bohórquez, Sandra C Correa Herrera ${ }^{3}$, Juan M Pardo Oviedo ${ }^{4}$, María Y Soracipa Muñoz , Ricardo Beltrán ${ }^{5}$, Gydnea L Aguirre Dávila ${ }^{6}$, Laura R Méndez Pino ${ }^{7}$.

\section{Resumen:}

Antecedentes: Con base en la teoría de la probabilidad y las proporciones de la entropía no equiprobable en el contexto de la teoría de los sistemas dinámicos se desarrolló una metodología de evaluación de la dinámica cardiaca. Objetivo: Confirmar la aplicabilidad clínica de la metodología diagnóstica basada en la probabilidad y la entropía para pacientes con diferentes tipos de arritmia evaluando el grado de agudización en la UCI por medio de sus proporciones características. Métodos: Se analizaron 80 registros Holter de la UCI con diagnóstico de arritmia y 20 normales. Se enmascararon las conclusiones clínicas del Holter y se tomaron de este los valores máximo, mínimo de frecuencia cardiaca y número total de latidos por hora para construir el atractor numérico para cada dinámica. Se calculó la probabilidad, la entropía, la relación $\mathrm{S} / \mathrm{k}$, y se evaluaron las proporciones de la entropía, estableciendo su diagnóstico matemático y cuantificando su nivel de gravedad. Resultados: Se demostró que la metodología diferencia en todos los casos dinámicas normales de anormales, obteniendo valores de sensibilidad y especificidad de $100 \%$ y coeficiente Kappa de 1, evidenciando que es posible además establecer cuantitativamente el grado de agudización. Conclusiones: La metodología desarrollada es útil en la práctica clínica para correlacionar la gravedad de las arritmias con predicciones de agudización físicas y matemáticas.

Palabras clave: diagnóstico; entropía; probabilidad; arritmias cardíacas.

\section{Abstract:}

Background: Based on the theory of probability and not equiprobable entropy ratios in the context of dynamical systems theory, a methodology for cardiac dynamic evaluation was developed. Objectives: To confirm the clinical applicability of the diagnostic methodology based on probability and entropy for patients with different types of arrhythmia assessing the degree of intensification in the ICU through its characteristic proportions. Methods: 80 Holter records from ICU diagnosed with arrhythmia and 20 normal were analyzed. Holter clinical findings were masked and were taken from them the maximum and minimum values of heart rate and total number of beats per minute to build the numerical attractor for each dynamics. Probability, entropy, S/k ratio, and proportions of entropy were evaluated, establishing its mathematical diagnosis and quantifying its severity level. Results: It was shown that the methodology difference in all cases normal of abnormal dynamics, obtaining a sensitivity and specificity of $100 \%$ and Kappa coefficient of 1 , showing that it is also possible to establish quantitatively the degree of exacerbation. Conclusions: The methodology developed is useful in clinical practice to correlate the severity of arrhythmias with physical and mathematical predictions of exacerbation.

Keywords: diagnosis; entropy; probability; arrhythmias; cardiac.

\footnotetext{
1 MD. Director del Grupo Insight. Director de la línea de profundización e internado especial física y matemáticas aplicadas a la medicina.

Universidad Militar Nueva Granada. Centro de Investigaciones Clínica del Country. Bogotá, Colombia.

2 Investigadora Grupo Insight. Universidad Militar Nueva Granada. Centro de investigaciones Clínica del Country. Bogotá, Colombia.

3 Psic. Investigadora Grupo Insight. Docente línea de profundización e internado especial física y matemáticas aplicadas a la medicina,

Universidad Militar Nueva Granada. Centro de Investigaciones Clínica del Country. Bogotá, Colombia.

4 MD. Gestor de conocimiento. Hospital Universitario Mayor Méderi. Bogotá, Colombia.

5 MD. Hospital Universitario Mayor Méderi. Bogotá, Colombia.

6 MD. Profesora facultad de medicina, Universidad Militar Nueva Granada.

7 Estudiante de la línea de profundización en Teorías físicas y matemáticas aplicadas a la medicina. Facultad de Medicina, Universidad Militar

Nueva Granada. Bogotá, Colombia.

Email de contacto: grupoinsight2025@yahoo.es
}

Recibido: 2017-01-11 Aceptado: 2017-05-09

DOI: 10.31053/1853-0605.v75.n1.16342 


\section{Introducción}

Teorías como la de los sistemas dinámicos, la probabilidad y la entropía, han sido utilizadas para la evaluación y diagnóstico del funcionamiento cardiaco. La teoría física que caracteriza el estado y la evolución de los sistemas es la teoría de los sistemas dinámicos ${ }^{1,2}$; la evolución se puede representar geométricamente en espacios abstractos denominados mapas de retardo, en los cuales se generan los atractores del sistema que pueden representar dinámicas predecibles -obteniendo atractores puntuales o cíclicos- o dinámicas impredecibles, representadas en atractores caóticos ${ }^{2,3}$.

El caos se subdivide en dos tipos determinista y estocástico. El primero presenta características tales como: la no linealidad del sistema, pérdida de precisión en la predicción, imposibilidad de establecer predicciones a largo plazo, conocimiento del comportamiento de las trayectorias y de sus propiedades estadísticas, cuando se presentan regularidades de este tipo ${ }^{4}$. El segundo se caracteriza por una duración nula de la memoria estadística ${ }^{5}$, al encontrarse sujeta a un proceso caótico resulta imposible determinar si el fenómeno es probable o improbable ${ }^{6}$.

La teoría de sistemas dinámicos y la geometría fractal constituyeron el fundamento para el desarrollo de una ley físico-matemática diagnóstica de aplicación clínica para los sistemas dinámicos cardiacos que diferencia normalidad, enfermedad aguda y evolución entre estos estados, cuya aplicación a pacientes con arritmia permitió establecer su nivel de gravedad e identificar casos con alteraciones leves en evolución hacia dinámicas patológicas que habían sido subdiagnosticadas hasta el momento ${ }^{7}$.

El lenguaje matemático que fundamenta la física moderna, se basa en la teoría de la probabilidad. Es una medida matemática que cuantifica la posible ocurrencia futura de un evento ${ }^{8}$. El concepto de entropía ha sido reinterpretado en otros contextos como la teoría cinética de los gases, la mecánica estadística y la teoría de la información ${ }^{9-11}$. En medicina ha sido utilizada como base para el desarrollo de medidas como la entropía aproximada, permitiendo caracterizar los cambios de la complejidad en diferentes sistemas ${ }^{12-14}$. Sin embargo su aplicabilidad clínica aún se encuentra en estudio ${ }^{13}$.

Dentro del contexto de los sistemas dinámicos, la teoría de la probabilidad y el establecimiento de proporciones de entropía, Rodríguez desarrollo diagnóstico matemático de la dinámica cardiaca en registros Holter, diferenciando normalidad, enfermedad crónica, enfermedad aguda y evolución entre estos estados. Se trata de una inducción matemática, basada en las frecuencias cardiacas máxima y mínima cada hora y el número de latidos/hora de10 registros Holter, a partir de los cuales se realizaron simulaciones computacionales para construir atractores numéricos de la dinámica por medio de la cuantificación de la frecuencia de aparición de los pares ordenados de las frecuencias cardiacas ${ }^{15}$.

Su capacidad diagnóstica y aplicabilidad clínica ha sido verificada en varios trabajos, incluyendo un estudio ciego de concordancia diagnóstica respecto al diagnóstico convencional aplicado a 450 pacientes normales y con diferentes patologías encontrando medidas de sensibilidad y especificidad del $100 \%$ y coeficiente Kappa igual a uno ${ }^{16}$. Adicionalmente se han realizado varias aplicaciones clínicas en la Unidad de Cuidados Intensivos $(\mathrm{UCI})^{17}$, logrando predecir procesos de agudización, así como el impacto de las intervenciones farmacológicas y quirúrgicas específicas en la dinámica cardiaca.

Entre las enfermedades cardiovasculares con mayor incidencia se encuentran las arritmias, que se asocian a un $50 \%$ de las muertes de origen cardiovascular ${ }^{18}$. Las arritmias son consideradas como alteraciones del ritmo cardiaco de diferente origen ${ }^{19}$ y se clasifican de acuerdo con la literatura médica en tres clases: pasivas, automáticas o ectópicas, y por reentrada. El diagnóstico convencional de las arritmias por parte del especialista, depende de variables implícitas en la historia clínica del paciente y del estudio de electrocardiograma ${ }^{20}$. Se considera un fenómeno complejo, que requiere la contemplación de diversos parámetros definidos para su detección, dificultando un diagnóstico objetivo y unívoco.

El propósito de la presente investigación es realizar una aplicación clínica de la metodología previamente desarrollada ${ }^{15}$, en casos normales y con arritmia de la $\mathrm{UCI}$ con el fin de cuantificar el nivel de gravedad y deterioro clínico de un paciente, evaluando si existe una correlación entre el grado de agudización físico matemático y la cantidad de eventos arrítmicos reportados clínicamente.

\section{Métodos}

\section{Definiciones}

Mapa de retardo: atractor específico que representa gráficamente la dinámica y la evolución de un sistema, ubicando pares ordenados de valores de una variable dinámica consecutiva en el tiempo en un espacio de dos o más dimensiones.

Par ordenado de frecuencias cardiacas: cada pareja de frecuencias consecutiva en el tiempo, denominada como $(\mathrm{X}, \mathrm{Y})$ y se ubican dentro de un mapa de retardo, en rangos de a cinco. 


\section{Ecuación 1: Probabilidad del rango $(X, Y)$}

$$
P(X, Y)=\frac{\text { Número de pares ordenados encontrados en el rango } \mathrm{X}, \mathrm{Y}}{\text { Total de pares ordenados del trazado }}
$$

Ecuación 2, Entropía del atractor: sistema no equiprobable, construido a partir de los rangos (X, Y), la entropía de ocupación de un atractor en el espacio de fases es calculada utilizando la siguiente ecuación.

$$
S=-K \sum_{x=1}^{n} \sum_{y=1}^{n} P(X, Y) \times \operatorname{Ln} P(X, Y)
$$

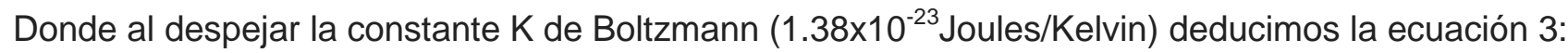

Ecuación 3, Proporción S/K:

$$
\frac{S}{K}=-\sum_{x=1}^{n} \sum_{y=1}^{n} P(X, Y) \times \operatorname{Ln} P(X, Y)
$$

Donde $S$ es la entropía $\mathrm{Y} P(\mathrm{X}, \mathrm{Y})$ es la probabilidad para cada rango $(\mathrm{X}, \mathrm{Y})$.Ecuación 3 que a su vez, puede ser reescrita de la siguiente forma:

Ecuación 3(a):

$$
\frac{S}{K}= \begin{cases}\sum_{U} P(U) \times \operatorname{Ln} P(U): & \operatorname{Unid} \operatorname{ades}(\mathrm{U}) \rightarrow(1-9) \\ \sum_{D} P(D) \times \operatorname{Ln} P(D): & \operatorname{Decenas}(\mathrm{D}) \rightarrow(10-99) \\ \sum_{C} P(C) \times \operatorname{Ln} P(C): & \text { Centenas }(\mathrm{C}) \rightarrow(100-999) \\ \sum_{M} P(M) \times \operatorname{Ln} P(M): & \text { Miles }(\mathrm{M}) \rightarrow(1000-9999)\end{cases}
$$

Esta ecuación al ser simplificada queda de la siguiente forma:

$$
\text { Ecuación 4: } \frac{S}{K}=T=U+D+C+M \text {; Donde } \mathrm{T}=\frac{S}{K}
$$

Proporciones entre las partes $(\mathrm{U}, \mathrm{D}, \mathrm{C}, \mathrm{M})$ y la totalidad $(\mathrm{T})$, que corresponde a la relación S/k.

\section{Regiones}

$$
U / T ; \quad D / T ; C / M ; M / T ; C / M \quad y \quad D / C
$$

Región 1: contiene todos los rangos de la frecuencia cardiaca que fueron comunes a todos los Holter normales. Región 2: contiene la totalidad de los rangos ocupados por Holter normales, excluyendo los de la región uno. Región 3: contiene los rangos de la frecuencia cardiaca que no son ocupados por los prototipos normales, es decir la región restante total del mapa de retardo.

\section{Procedimiento de recolección y análisis de información}

Se analizaron en total 100 registros Holter con edad mayor a 21 años, de los cuales 80 provenían de la UCI del Hospital Universitario Mayor y tenían diagnóstico de arritmia y 20 correspondían a registros de sujetos normales provenientes de las bases de datos del Grupo Insight. De los Holter normales, 5 corresponden a pacientes que se realizaron este examen por indicación médica ante alguna sintomatología específica, pero los resultados del Holter se encontraron dentro de los límites normales; mientras que los 15 restantes corresponden a casos de control, provenientes de sujetos sin sintomatologías ni patologías cardiacas previas. La interpretación diagnóstica del Holter fue realizada por 
cardiólogos o electrofisiólogos expertos de la institución de acuerdo con los parámetros de evaluación clínica convencional.

La información clínica y conclusiones registrados en cada Holter fueron enmascarados (tabla 1). La única información no enmascarada correspondió a los valores para cada hora de frecuencias mínimas y máximas y el número de latidos. A partir de estos valores y con base en la metodología desarrollada previamente por Rodríguez ${ }^{15}$ se tomaron los valores de frecuencias máximas, mínimas y el número de latidos cada hora, se simularon los valores consecutivos de las frecuencias cardiacas en un software previamente diseñado para esta metodología. Seguidamente se construyó un mapa de retardo en el cual se graficó un atractor numérico con los valores hallados, en el cual, en lugar de los puntos unidos por una línea continua que constituyen los atractores caóticos convencionales, se representa el número de frecuencias de los pares ordenados de la frecuencia cardiaca en rangos de a cinco lat. /min que se encuentra en cada lugar del atractor.

Seguidamente para cada una de las regiones evaluadas se calculó la probabilidad de aparición de los pares ordenados de frecuencias cardiacas respecto a la totalidad, para cada uno de los registros en los rangos de a cinco en el espacio de fases. Posteriormente se evaluó la entropía de cada atractor (ecuación 2) y se calculó la relación S/k (ecuación 3).

A continuación se agruparon los valores hallados para cada uno de los sumandos según sea el valor en la ecuación 3(a), los cuales corresponden a probabilidades asociadas a frecuencias de ocupación en unidades, decenas, centenas y miles. El desarrollo dichas sumas permite evaluar las proporciones existentes entre cada sumando respecto a la totalidad, que corresponde a la proporción S/k, así como las proporciones entre centenas respecto a miles y decenas respecto a centenas para cada región determinada.

El criterio para diferenciar normalidad y anormalidad para cada Holter, establecido previamente por Rodríguez ${ }^{15,16}$ se hizo mediante el análisis del número de proporciones, en cualquiera de las tres regiones, que se encuentre fuera de los límites de normalidad definidos. Según este criterio, si hay dos o más valores fuera de estos rangos la dinámica es anormal, y en caso contrario la dinámica es diagnosticada como normal.

Para cuantificar el nivel de gravedad de la dinámica patológica se evaluó cuantitativamente la distancia de la dinámica respecto a la normalidad, mediante restas entre los valores de las proporciones de la dinámica que se encuentren por fuera de los límites de normalidad y los valores de los extremos de los intervalos de normalidad. Es decir, si el valor es mayor al intervalo definido para normalidad se le resta el valor límite superior de normalidad, mientras que si el valor es inferior a este intervalo, es restado del valor límite inferior. El valor de las restas halladas se agrupó suma según el orden de magnitud de sus frecuencias de aparición, y los valores de cada grupo fueron del orden de unidades (U), decenas (D), centenas $(\mathrm{C})$ y miles $(\mathrm{M})$.

Para la cuantificación de un estado más crítico o agudo de la dinámica, se analiza el valor de las sumas de las restas agrupadas. En el caso del orden de miles, en un estudio realizado en la UCI se evidencio que cuando este valor aumenta se correlaciona con un paciente que presenta deterioro clínico o un estado de agudización, por ejemplo, un Infarto agudo de Miocardio. Por el contrario, una disminución de estos valores correspondió a una evolución satisfactoria de un paciente sometido a una intervención quirúrgica $^{15}$.

\section{Análisis estadístico}

Para el análisis estadístico se incluyeron los 15 holter normales sin sintomatologías previas y los 80 holter de arritmias provenientes de UCI. Las conclusiones clínicas del registro Holter fueron desenmascaradas y el diagnóstico emitido según parámetros médicos convencionales se asumió como Gold estándar para compararlo con el diagnóstico físico-matemático. Se procedió a calcular la sensibilidad, especificidad, cantidad de falsos negativos y falsos positivos a través de una tabla de contingencia $2 * 2$. Finalmente, se procedió a evaluar mediante el coeficiente Kappa la concordancia diagnóstica entre el Gold estándar y el diagnóstico físico-matemático.

\section{Resultados}

Se encontró que los valores de entropía de los 100 atractores variaron entre 4,92E-23 y 7,84E-23; entre 6,47E-23 y 7,01E-23 para los 15 Holter normales sin sintomatologías o patologías previas, entre 6,42E23 y 6,98E-23 para los 5 holter con algún tipo de sintomatología, y entre 4,92E-23 y 7,84E-23 para los de UCI. Los holter normales sin sintomatologías o patologías previas presentaron proporciones de la 
entropía para la región 1 entre 0 y 2,761, para la región 2 entre 0 y 3,166, y para la región 3 presentaron valores de 0 en todos los casos. Los holter normales con sintomatologías presentaron proporciones de la entropía para la región 1 entre 0 y 3,132, para la región 2 entre 0 y 8,892, y para la región 3 presentaron siempre valores de 0 . Los holter de $\mathrm{UCl}$ presentaron proporciones de la entropía para la región 1 entre 0 y 6,354; para la región 2 entre 0 y 2,603 y para la región 3 entre 0 y 4,408. Ver Tabla 2. Todos los holter normales sin sintomatologías o patologías previas presentaron proporciones de la entropía dentro de los límites de normalidad establecidos previamente ${ }^{15}$, mientras que los holter con alguna sintomatología presentaron en cuatro de los cinco casos dos o más valores por fuera de estos límites, y los Holter de UCI presentaron en todos los casos por lo menos dos proporciones por fuera de los límites de normalidad, estableciéndose de este modo su diagnóstico patológico desde la metodología físico-matemática.

\begin{tabular}{|c|c|c|c|c|}
\hline & SEXO & EDAD & INDICACIONES & CONCLUSIONES \\
\hline $\mathrm{N} 1$ & $\mathrm{~F}$ & 35 & Caso de control & FC prom 75; FCmín 57 a 04:34; FC máx 136 a 16:01. Estudio dentro de límites normales. \\
\hline $\mathrm{N} 2$ & M & 26 & Caso de control & FC prom 72; FCmín 59 a 02:53; FC máx 141 a 12:17. Estudio dentro de límites normales. \\
\hline $\mathrm{N} 3$ & M & 49 & Síncope & FC prom 84; FCmín 60 a 05:41; FC máx 156 a 20:40. Estudio dentro de límites normales. \\
\hline 1 & $\mathrm{~F}$ & 84 & Bradiarritmia & $\begin{array}{l}\text { Ritmosinusal. FC prom } 63 \mathrm{lpm}, \mathrm{FC} \text { min } 50, \mathrm{FC} \text { max 100. No arritmias ventriculares. Ocasionales ectopias } \\
\text { atriales con algunas dupletas y dos salvas de tres y cuatro }\end{array}$ \\
\hline 2 & $\mathrm{~F}$ & 58 & arritmia cardiaca & $\begin{array}{l}\text { Fibrilacion atrial con respuesta ventricular no controlada: FC prom 103, FC min } 67 \text { lpmFcmax 153. } 37 \\
\text { ectopias ventriculares monomorfas, } 2 \text { dupletas }\end{array}$ \\
\hline 3 & M & 74 & arritmia cardiaca & $\begin{array}{l}\text { Ritmo sinusal. Fcprom } 65 \mathrm{lpm}, \mathrm{Fc} \min 46 \mathrm{lpm}, \mathrm{Fcmax} 96 \mathrm{lpm} .28911 \text { ectopias ventriculares monomorfas, } \\
78 \text { episodios de taquicardias ventricular no sostenida, frecuentes dupletas (4637), } 871 \text { de eventos de } \\
\text { bigeminismo y } 292 \text { de eventos de trigeminismo. QRS ancho con imagen en favor de bloqueo de rama } \\
\text { derecha. }\end{array}$ \\
\hline 4 & M & 80 & Estudio arritmia & $\begin{array}{l}\text { Ritmo sinusal FC prom } 63 \mathrm{lpm}, \mathrm{FC} \text { min } 51 \mathrm{lpm} \text { FC max. } 55 \text { ectopias ventriculares monomorfas con } \\
\text { algunas dupletas (3), indice de prematuridad > } 1 \text { y dos eventos de taquicardia ventricular. ectopias atriales } \\
\text { (38 ESV), fibrilacion atrial de } 3 \text { segs. Variabilidad RR ligeramente disminuida con un SDNN de } 89\end{array}$ \\
\hline 5 & M & 78 & estudio arritmia & $\begin{array}{l}\text { Ritmo sinusal FC prom } 78 \mathrm{lpm}, \mathrm{FC} \text { min } 64 \mathrm{lpm} \text {, FC max. } 550 \text { ectopias ventriculares dimorfas, dupletas y } \\
\text { trigeminismo. ectopias atriales ( } 422 \mathrm{ESV} \text { ) con dupletas y salvas de taquicardia atrial. Variabilidad RR } \\
\text { disminuida con un SDNN de } 70 \mathrm{~ms} \text {. }\end{array}$ \\
\hline 6 & M & 33 & Sincope & $\begin{array}{l}\text { Arritmia sinusal. Fcprom } 66 \text { lpm, Fc min } 43 \text { lpm, Fcmax 127. QRS con morfologia en favor de bloqueo de } \\
\text { rama derecha }\end{array}$ \\
\hline 7 & $\mathrm{~F}$ & 83 & $\begin{array}{l}\text { Infarto Agudo de } \\
\text { Miocardio }\end{array}$ & $\begin{array}{l}\text { Ritmo sinusal FC prom } 60 \mathrm{lpm}, \mathrm{FC} \min 41 \mathrm{lpm} \text { FC max } 90 \mathrm{lpm} \text {. } 3060 \text { ectopias ventriculares monomorfas, } \\
\text { aisladas, intercaladas, taquicardia ventricular no sostenida. } 34 \text { dupletas ventriculares, eventos de } \\
\text { trigeminismo. Ectopias atriales aisladas, Variabilidad RR conservada. }\end{array}$ \\
\hline 8 & $\mathrm{~F}$ & 75 & $\begin{array}{l}\text { Enfermedad del } \\
\text { nodo }\end{array}$ & $\begin{array}{l}\text { Ritmo sinusal, FC prom, fue de } 50 \mathrm{lpm}, \mathrm{FC} \min 26 \mathrm{lpm}, \mathrm{FC} \max \text { de } 92 \mathrm{lpm} \text {. Ritmos de escape de la union, } \\
3622 \text { ectopias ventriculares dimorfas, } 28 \text { dupletas bidireccionales, } 157 \text { bigeminismo y } 194 \text { trigeminismo. }\end{array}$ \\
\hline 9 & $\mathrm{~F}$ & 75 & Sincope & $\begin{array}{l}\text { Ritmo sinusal. Fc } 67 \mathrm{lpm}, \mathrm{Fc} \text { min } 47 \mathrm{lpmFcmax} 125 \mathrm{lpm} \text {. } 1234 \text { ectopias ventriculares monomorfas, } 7 \\
\text { dupletas y } 7 \text { de eventos de trigeminismo. Ritmos de la union en favor de enfermedad del nodo. episodio de } \\
\text { bloqueo AV. }\end{array}$ \\
\hline 10 & M & 60 & $\begin{array}{l}\text { Cardiopatía } \\
\text { isquémica. } \\
\text { Bloqueo } \\
\text { trifasicular }\end{array}$ & $\begin{array}{l}\text { Ritmo sinusal. FC prom } 68 \text { lpm, Fc min } 51 \text { lpmFcmax } 98 \text { lpm. } 6228 \text { ectopias ventriculares monomorfas, } \\
10 \text { duplas, } 5 \text { de eventos de bigeminismo y } 236 \text { de eventos de trigeminismo, Bloqueo AV de primer grado } \\
\text { con un PR de hasta } 280 \mathrm{~ms} \text {. Variabilidad RR ligeramente disminuida con un SDNN de } 98 \mathrm{~ms} \text {. }\end{array}$ \\
\hline 11 & $\mathrm{~F}$ & 87 & Sincope & $\begin{array}{l}\text { Ritmosinusal. Fc prom } 68 \mathrm{lpm}, \mathrm{Fc} \min 54 \mathrm{lpm}, \mathrm{Fc} \text { max } 92 \text {. ectopias ventriculares monomorfas escasas, } \\
\text { un episodio de taquicardia ventricular. Variabilidad RR severamente disminuida con un SDNN de } 61 \mathrm{~ms} \text {. }\end{array}$ \\
\hline 12 & $\mathrm{~F}$ & 71 & $\begin{array}{l}\text { fibrilación } \\
\text { auricular }\end{array}$ & $\begin{array}{l}\text { fibrilacion atrial permanente con respuesta ventricular controlada: Fcprom } 81 \mathrm{lpm}, \mathrm{Fc} \text { min } 47 \mathrm{lpm}, \mathrm{Fcmax} \\
151 \mathrm{lpm} \text {. Ectopias ventriculares muy poco frecuentes. un evento de taquicardia ventricular. }\end{array}$ \\
\hline 13 & M & 68 & Bradicardia & $\begin{array}{l}\text { Ritmo sinusal con tendencia a la bradicardia: Fcprom 50, Fc min } 41 \mathrm{lpm}, \text { Fcmax } 69 \mathrm{lpm} \text {. } 235 \text { ectopias } \\
\text { ventriculares monomorfas aisladas. Ocasionales ectopias atriales con algunas dupletas y una salva de } \\
\text { taquicardia atrial. Variabilidad RR ligeramente disminuida con un SDNN de } 95 \mathrm{~ms} \text {. }\end{array}$ \\
\hline 14 & $\mathrm{~F}$ & 78 & Sincope & $\begin{array}{l}\text { Ritmo sinusal. Fcprom } 64 \mathrm{lpm}, \mathrm{Fc} \min 48 \mathrm{lpm} \text {, Fcmax } 112 \mathrm{lpm} \text {. } 1678 \text { Ectopias ventriculares monomorfas } \\
\text { con una dupleta, Ocasionales ectopias atriales ( } 306 \mathrm{ESV} \text { ) aisaldas, algunas dupletas. bloqueo de rama } \\
\text { izquierda permanente. Variabilidad RR disminuida con un SDNN de } 89 \mathrm{~ms}\end{array}$ \\
\hline
\end{tabular}


ENTROPÍA PROPORCIONAL APLICADA A LA UCI.

\begin{tabular}{|c|c|c|c|c|}
\hline 15 & $\mathrm{~F}$ & 80 & Taquicardia & $\begin{array}{l}\text { Ritmo sinusal. Fcprom } 99 \mathrm{lpm}, \mathrm{Fc} \text { min } 64 \mathrm{lpm}, \text { Fcmax } 139 \mathrm{lpm} \text {. } 5569 \text { Ectopias ventriculares dimorfas con } \\
\text { algunas dupletas (95), y un episodio de taquicardia ventricular polimorfica, } 83 \text { de eventos de bigeminismo } \\
\text { y } 44 \text { de eventos de trigeminismo. Variabilidad RR disminuida con un SDNN de } 71 \mathrm{~ms} \text {. }\end{array}$ \\
\hline 16 & M & 81 & $\begin{array}{l}\text { Infarto Agudo de } \\
\quad \text { Miocardio }\end{array}$ & $\begin{array}{l}\text { Ritmo sinusal.Fcprom } 91 \mathrm{lpm}, \mathrm{Fc} \text { min } 70 \mathrm{lpm} \text {, Fcmax } 122 \mathrm{lpm} .1751 \text { ectopias ventriculares monomorfas, } \\
7 \text { dupletas y } 52 \text { trigeminismo. numerosas ectopias atriales ( } 5323 \mathrm{ESV} \text { ), dupletas. Variabilidad RR } \\
\text { severamente disminuida con un SDNN de } 47 \mathrm{~ms} \text {. }\end{array}$ \\
\hline 17 & $\mathrm{M}$ & 72 & Falla cardiaca & $\begin{array}{l}\text { Ritmosinusal.Fc prom } 87 \mathrm{lpm}, \mathrm{Fc} 66, \mathrm{Fc} \text { max 104. } 3501 \text { Ectopias ventriculares Dimorfas, } 2 \text { bigeminismo y } \\
38 \text { trigeminismos, algunas dupletas, dos Taquicardia ventricular lenta. Variabilidad RR severamente } \\
\text { disminuida con un SDNN de } 44 \mathrm{~ms}\end{array}$ \\
\hline 18 & M & 82 & Lipotimia & $\begin{array}{l}\text { fibrilacion Atrial. Feprom } 60 \mathrm{lpm}, \mathrm{Fc} \text { min } 31 \mathrm{lpm}, \mathrm{Fcmax} 103 \mathrm{lpm} \text {. } 2210 \text { ectopias ventriculares } \\
\text { monomorfas, } 3 \text { taquicardia ventricular y } 132 \text { dupletas, } 1 \text { bigeminismo y } 7 \text { trigeminismo }\end{array}$ \\
\hline 19 & $\mathrm{~F}$ & 79 & Sincope & $\begin{array}{l}\text { Ritmosinusal.Fc prom } 96 \text { lpm, Fc min 81, Fc max 126. Variabilidad RR severamente disminuida con un } \\
\text { SDNN de } 46 \mathrm{~ms}\end{array}$ \\
\hline 20 & $\mathrm{~F}$ & 85 & Dolor torácico & $\begin{array}{l}\text { Ritmosinusal. Fc prom } 74 \text { lpm, Fc min } 56 \text { lpm, Fc max 142. } 1133 \text { ectopias ventriculares monomorfas, con } \\
\text { algunas dupletas }\end{array}$ \\
\hline 21 & $\mathrm{~F}$ & 66 & $\begin{array}{l}\text { Enfermedad } \\
\text { chagasica }\end{array}$ & $\begin{array}{l}\text { Ritmo de base en fibrilacion auricular con respuesta lenta. Extrasistolia ventricular muy frecuente, } \\
\text { polimorfa con dupletas. Trastorno de conducción intraventricular por bloqueo de rama }\end{array}$ \\
\hline
\end{tabular}

N1, N2: dinámicas normales sin sintomatologías, y N3: dinámica normal con sintomatologías.

Al realizar el análisis de las restas de los valores de las proporciones que se encontraban por fuera de los límites de normalidad, respecto a dichos límites, agrupadas y sumadas de acuerdo al orden de magnitud de su frecuencia correspondiente, se encontró que dado que los holter normales sin sintomatologías previas presentaron siempre valores dentro de los límites de normalidad, todos sus valores son iguales a cero. En contraposición, los holter normales que presentaron sintomatologías presentaron valores de Miles de 0 , de Centenas entre 0 y 1,651, de Decenas entre 0 y 0,132 y de Unidades entre 0 y 0,426 . Por otro lado, los valores de Miles del grupo de $\mathrm{UCl}$ se encontraron entre 0 y 3,905; para las Centenas se encontraron valores entre 0,317 y 5,873; para las Decenas se encontraron valores entre 0 y 0,001 y para las Unidades se encontraron valores entre 0 y 0,077.

\begin{tabular}{|c|c|c|c|c|c|c|c|c|c|c|c|c|c|c|c|c|c|c|c|c|c|c|c|c|c|}
\hline & & \multicolumn{3}{|c|}{ NORMALES } & \multirow{2}{*}{\multicolumn{21}{|c|}{ HOLTER DE UCI CON ARRITMIAS CARDIACAS }} \\
\hline & & \multicolumn{2}{|c|}{ ss } & \multirow{2}{*}{$\begin{array}{l}\text { CS } \\
\mathrm{N} 3\end{array}$} & & & & & & & & & & & & & & & & & & & & & \\
\hline & & N1 & $\mathrm{N} 2$ & & 1 & 2 & 3 & 4 & 5 & 6 & 7 & 8 & 9 & 10 & 11 & 12 & 13 & 14 & 15 & 16 & 17 & 18 & 19 & 20 & 21 \\
\hline & $\mathrm{S} / \mathbf{k}$ & 4,741 & 5,03 & 4,872 & 4,694 & 5,456 & 5,052 & 4,219 & 4,808 & 4,964 & 4,85 & 5,212 & 4,87 & 4,17 & 3,79 & 5,683 & 3,566 & 4,805 & 4,724 & 4,595 & 4,115 & 5,105 & 3,836 & 5,059 & 4,555 \\
\hline & intropía & 6,50 & 6,90 & 6,70 & 6,50 & 7,50 & 7,00 & 5,80 & 6,60 & 6,90 & 6,70 & 7,20 & 6,70 & 5,80 & 5,20 & 7,80 & 4,90 & 6,60 & 6,50 & 6,30 & 5,70 & 7,00 & 5,30 & 7,00 & 6,30 \\
\hline & $\mathrm{U} / \mathrm{T}$ & 0 & 0 & 0 & 0,002 & 0 & 0,003 & 0,001 & 0 & 0,002 & 0 & 0 & 0 & 0 & 0 & 0 & 0,032 & 0,002 & 0 & 0,001 & 0 & 0 & 0 & 0 & 0 \\
\hline & $\bar{D} / \mathrm{T}$ & 0,088 & 0,092 & 0,005 & 0,024 & 0,009 & 0,025 & 0,069 & 0,056 & 0,026 & 0,023 & 0,01 & 0,044 & 0,011 & 0,057 & 0,019 & 0,022 & 0,053 & 0,016 & 0,022 & 0,011 & 0,034 & 0,011 & 0,063 & 0,025 \\
\hline & $\mathrm{C} / \mathrm{T}$ & 0,483 & 0,551 & 0,477 & 0,408 & 0,695 & 0,785 & 0,219 & 0,418 & 0,393 & 0,653 & 0,413 & 0,278 & 0,261 & 0,217 & 0,672 & 0,059 & 0,264 & 0,246 & 0,176 & 0,18 & 0,553 & 0,185 & 0,448 & 0,199 \\
\hline & $\mathrm{M} / \mathrm{T}$ & 0,309 & 0,176 & 0,428 & 0,45 & 0 & 0 & 0,648 & 0,432 & 0,415 & 0,103 & 0 & 0,459 & 0,692 & 0,702 & 0 & 0,275 & 0,534 & 0,713 & 0,73 & 0,798 & 0 & 0,803 & 0,374 & 0 \\
\hline & $\mathrm{C} / \mathrm{M}$ & 1,566 & 3,12 & 1,114 & 0,905 & & & 0,339 & 0,968 & 0,946 & 6,354 & & 0,605 & 0,376 & 0,309 & & 0,212 & 0,494 & 0,344 & 0,242 & 0,225 & & 0,231 & 1,2 & \\
\hline & $\mathrm{D} / \mathrm{C}$ & 0,183 & 0,167 & 0,011 & 0,059 & 0,012 & 0,032 & 0,314 & 0,134 & 0,067 & 0,035 & 0,024 & 0,16 & 0,041 & 0,263 & 0,028 & 0,377 & 0,2 & 0,066 & 0,125 & 0,061 & 0,062 & 0,061 & 0,141 & 0,128 \\
\hline & $\mathrm{U} / \mathrm{T}$ & 0,008 & 0,008 & 0,001 & 0,001 & 0,001 & 0,001 & 0 & 0,001 & 0,001 & 0 & 0 & 0 & 0 & 0 & 0,002 & 0,033 & 0,013 & 0 & 0 & 0 & 0 & 0 & 0,001 & 0 \\
\hline & $\mathrm{D} / \mathrm{T}$ & 0,084 & 0,132 & 0,042 & 0,023 & 0,034 & 0,011 & 0,028 & 0,055 & 0,017 & 0,009 & 0,003 & 0,025 & 0,002 & 0,022 & 0,065 & 0,004 & 0,058 & 0,014 & 0,005 & 0,003 & 0,017 & 0 & 0,025 & 0,005 \\
\hline & $\mathrm{C} / \mathrm{T}$ & 0,027 & 0,04 & 0,047 & 0,069 & 0,261 & 0,116 & 0,026 & 0,031 & 0,096 & 0,139 & 0,098 & 0,09 & 0,034 & 0 & 0,165 & 0 & 0,047 & 0,01 & 0,048 & 0,008 & 0,127 & 0 & 0,071 & 0,111 \\
\hline & $\mathrm{M} / \mathrm{T}$ & 0 & 0 & 0 & 0 & 0 & 0 & 0 & 0 & 0 & 0 & 0 & 0 & 0 & 0 & 0 & 0,341 & 0 & 0 & 0,018 & 0 & 0 & 0 & 0 & 0 \\
\hline & $\mathrm{C} / \mathrm{M}$ & & & & & & & & & & & & & & & & 0 & & & 2,603 & & & & & \\
\hline & $\mathrm{D} / \mathrm{C}$ & & 3,29 & 0,892 & 0,333 & 0,132 & 0,098 & 1,07 & 1,769 & 0,177 & 0,063 & 0,032 & 0,282 & 0,059 & & 0,395 & & 1,235 & 1,393 & 0,1 & 0,449 & 0,133 & & 0,357 & 0,049 \\
\hline & $\mathrm{U} / \mathrm{T}$ & 0 & 0 & 0 & 0,001 & 0 & 0,001 & 0,001 & 0 & 0 & 0,001 & 0,002 & 0,001 & 0 & 0 & 0 & 0,022 & 0,003 & 0 & 0 & 0 & 0,001 & 0 & 0,001 & 0 \\
\hline & $\mathrm{D} / \mathrm{T}$ & 0 & 0 & 0 & 0,01 & 0 & 0,019 & 0,007 & 0,007 & 0,015 & 0,015 & 0,034 & 0,024 & 0 & 0,001 & 0,016 & 0,015 & 0,021 & 0 & 0 & 0 & 0,026 & 0 & 0,017 & 0,032 \\
\hline & $\mathrm{C} / \mathrm{T}$ & 0 & 0 & 0 & 0,012 & 0 & 0,039 & 0 & 0 & 0,035 & 0,058 & 0,439 & 0,078 & 0 & 0 & 0,061 & 0,075 & 0,005 & 0 & 0 & 0 & 0,241 & 0 & 0 & 0,627 \\
\hline & $\mathrm{M} / \mathrm{T}$ & 0 & 0 & 0 & 0 & 0 & 0 & 0 & 0 & 0 & 0 & 0 & 0 & 0 & 0 & 0 & 0,124 & 0 & 0 & 0 & 0 & 0 & 0 & 0 & 0 \\
\hline & $\mathrm{C} / \mathrm{M}$ & & & & & & & & & & & & & & & & 0,603 & & & & & & & & \\
\hline & $\mathrm{D} / \mathrm{C}$ & & & & 0,851 & & 0,499 & & & 0,412 & 0,26 & 0,078 & 0,314 & & & 0,265 & 0,2 & 4,408 & & & & 0,107 & & & 0,051 \\
\hline \multirow{4}{*}{$\sum_{\infty}^{\pi}$} & $\mathbf{U}$ & 0 & 0 & 0 & 0,003 & 0 & 0,004 & 0,002 & 0 & 0,002 & 0,001 & 0,002 & 0,001 & 0 & 0 & 0 & 0,077 & 0,01 & 0 & 0 & 0 & 0,001 & 0 & 0,001 & 0 \\
\hline & D & 0 & 0 & 0,034 & 0,025 & 0,03 & 0,033 & 0,007 & 0,007 & 0,027 & 0,031 & 0,063 & 0,024 & 0,028 & 0,001 & 0,036 & 0,032 & 0,021 & 0,023 & 0,017 & 0,028 & 0,03 & 0,029 & 0,017 & 0,046 \\
\hline & C & 0 & 0 & 1,651 & 3,116 & 2,761 & 3,257 & 1,778 & 0,756 & 2,889 & 2,961 & 3,129 & 2,824 & 2,664 & 0,338 & 2,688 & 0,886 & 5,873 & 1,319 & 2,674 & 2,334 & 2,782 & 0,317 & 2,168 & 3,444 \\
\hline & M & & & 0 & 0,107 & 0,153 & 0,153 & 0,844 & 0,043 & 0,066 & 2,797 & 0,153 & 0,407 & 0,851 & 0,928 & 0,153 & 3,905 & 0,575 & 0,904 & 1,568 & 1,108 & 0,153 & 1,107 & 0 & 0,153 \\
\hline
\end{tabular}

SS: dinámicas normales sin sintomatologías, CS: dinámica normal con sintomatologías, Proporciones de la entropía para cada región; U/T: Unidades/ Totalidad, D/T:

Decenas/ Totalidad, C/T: Centenas/ Totalidad, M/T: Miles/Totalidad, C/M: Centenas/Miles, D/C: Decenas/ Centenas

La teoría predice que las dinámicas cardiacas que presenten valores más altos en las sumas de las restas de Miles, corresponden a los estados más agudos de acuerdo con las observaciones clínicas, mientras que los valores menores corresponden a casos menos severos, lo cual fue corroborado con las observaciones clínicas. Ver tablas 1 y 2. 
Como resultado del análisis estadístico, se determinó que los valores para la especificidad y sensibilidad de los Holter evaluados fueron del 100\% al comparar el diagnóstico matemático con el Gold standard, de la misma manera el valor correspondiente a la concordancia entre el diagnóstico físico matemático y el diagnóstico clínico convencional, determinado mediante el coeficiente Kappa fue igual a 1.

\section{Discusión}

Este es el primer trabajo en el que se confirma la capacidad diagnóstica y predictiva de la metodología de evaluación de la dinámica cardiaca basada en la probabilidad y proporciones de la entropía en casos normales y con arritmias provenientes de la UCl cuantificando el nivel de agudización de las dinámicas cardiacas arrítmicas y demostrando la correlación de las predicciones físico-matemáticas de agudización con el diagnóstico clínico convencional.

El análisis estadístico mostró que la metodología fue capaz de diferenciar objetiva y cuantitativamente dinámicas normales de arrítmicos. Así mismo, se corroboró que las dinámicas arrítmicas que exhibieron mayores valores en las sumas de las restas del orden de miles correspondieron a casos de la $\mathrm{UCl}$ en estados agudos o críticos, confirmando los hallazgos previos en los que se realizan aplicaciones de esta metodología a Holter de $\mathrm{UCl}^{17}$. Se hizo el análisis estadístico de concordancia diagnóstica con el fin de cumplir con los estándares de la investigación médica actual, y por su utilidad para el análisis de una patología específica, como la arritmia cardiaca.

Por ejemplo, el Holter No. 7, que presentó un valor de 2,797 en los valores de Miles y 2,961 en Centenas, corresponde al Holter de un paciente con Infarto Agudo de Miocardio que presentó múltiples eventos arrítmicos, incluyendo 3060 ectopias ventriculares monomorfas. Cabe resaltar que aunque presentó un valor matemático característico de un estado severamente agudo, presentó una Variabilidad $\mathrm{RR}$ conservada, lo que es un indicador de las dificultades de las medidas de la variabilidad para cuantificar el nivel de gravedad de un estado agudo. En contraposición, el Holter No. 5, que presentó un valor de Miles de 0,043, mucho menores que el anterior, aunque presentó características típicas de arritmia de acuerdo con la evaluación convencional, presentó características clínicas mucho menos severas que las del caso anterior, con 550 ectopias ventriculares dimorfas, y 422 ESV. Ver tablas 1 y 2. Ambas dinámicas presentan cuadros agudos desde la evaluación clínica convencional, sin embargo el segundo es mucho menos severo que el primero, lo que corresponde con las cuantificaciones matemáticas, donde el valor de Miles del primer caso es mucho mayor que el del segundo, confirmando las predicciones. Ver tablas 1 y 2.

Con base en la metodología, los órdenes de magnitud de los valores de miles permiten determinar el grado de severidad de la dinámica, los valores de las centenas, decenas y unidades también dan cuenta de las diferencias entre ellas. Por ejemplo, las dinámicas No 12 y No 21 presentan en ambos casos un valor de miles de 0,153, sin embargo el valor de centenas del No 12 es de 2,688, mientras que el del No 21 es de 3,444. La teoría predice que el No 12 presenta un estado clínico menos severo que el No 21, lo cual fue corroborado al comparar las conclusiones del Holter, donde para el No 12 se reporta fibrilación atrial permanente con respuesta ventricular controlada con ectopias ventriculares muy poco frecuentes y un evento de taquicardia ventricular; en contraposición, el No 21 corresponde a un caso de enfermedad chagásica con un cuadro más severo que el primero, al presentar un trastorno de conducción intraventricular por bloqueo de rama, con ritmo de base en fibrilación auricular con respuesta lenta, que incluye extrasistolias ventriculares muy frecuentes, polimorfas y con dupletas. Ver tablas 1 y 2.

La dinámica no. 1 presentó un valor mayor de miles de todos los evaluados, denotando una dinámica en un proceso severamente agudo; sin embargo las conclusiones convencionales del Holter, aunque evidencian un estado de bradicardia, no dan cuenta de este estado de agudización. Este caso sirve como ejemplo de la forma en que la evaluación físico-matemática de la autoorganización total del sistema permite predecir procesos de agudización no son perceptibles desde las medidas convencionales de cuantificación de eventos arrítmicos.

Adicionalmente el análisis de los holter clasificados dentro de los límites de normalidad pero que reportaron sintomatologías previas, presentaron en cuatro de los cinco casos valores por fuera de los límites de normalidad, en contraposición con los Holter normales sin sintomatologías previas, que en todos los casos fueron diagnosticados como normales. Este resultado señala la capacidad de la metodología para establecer medidas que alertan sobre alteraciones leves subdiagnosticadas, que pueden dar lugar a estados patológicos, y que pueden requerir un seguimiento clínico más específico en el tiempo. Se evidencia además que presentaron valores de miles de cero en todos los casos, señalando su carácter mucho menos severo, comparado con los Holter de UCI 
Con base en la teoría de los sistemas dinámicos no lineales se ha evidenciado que el comportamiento cardiaco característico de normalidad contradice la premisa clásica de regularidad homeostática ${ }^{22}$, al demostrar que la dinámica patológica se caracteriza por comportamientos excesivamente regulares 0 excesivamente aleatorios, mientras que la normalidad se encuentra dentro de estos dos extremos ${ }^{23}$. Desde esta perspectiva, se han establecido diferentes tipos de medida para la caracterización y predicción de eventos anormales en el sistema dinámico cardiaco ${ }^{24-26}$. Sin embargo, a pesar de los avances logrados, aún no puede afirmarse su aplicabilidad clínica ${ }^{26}$.

El estudio de la dinámica cardiaca desde una perspectiva físico-matemática estricta, hace innecesario el uso de las consideraciones de tipo estadístico que fundamentan dichas metodologías, aclarando como un valor cuantitativo permite establecer diferencias entre una y otra arritmia, aun cuando presenten características muy similares desde la perspectiva clínica convencional, constituyendo una herramienta práctica para la toma de decisiones a nivel clínico.

Esta nueva aproximación, plantea una evaluación de la entropía informacional en un contexto geométrico que permite evidenciar un orden físico acausal subyacente de carácter predictivo, al margen de la aleatoriedad y azar asociados normalmente a la teoría del caos y los sistemas dinámicos no lineales. El contexto acausal ha sido el fundamento de teorías de la física como la teoría del $\operatorname{caos}^{3,9-15,27}$, la mecánica cuántica ${ }^{28}$ y la mecánica estadística ${ }^{10-11}$. Trabajos en otras áreas de la medicina han desarrollado metodologías basadas en probabilidad y entropía, que evidencian y establecen una autoorganización de diferentes sistemas con carácter predictivo, ya sea en inmunología ${ }^{29}$, predicción de epidemias ${ }^{30}$ dinámica cardiaca fetal ${ }^{31}$.Otras teorías físicas y matemáticas han logrado resultados diagnósticos predictivos en otros fenómenos de la medicina, como la morfología celular ${ }^{32} \mathrm{y} \operatorname{arterial}^{33}$, la infectología ${ }^{34}$, también se han establecido predicciones de mortalidad en la Unidad de Cuidados Intensivos ${ }^{35}$, mostrando que con la aplicación de teorías físicas y matemáticas es posible hallar un orden en diferentes fenómenos que se perciben como aleatorios o indeterminados, evidenciando que existen órdenes físico-matemáticos subyacentes a los diferentes fenómenos médicos, cuyo establecimiento permite mejorar los diagnósticos y métodos predictivos actuales.

\section{Conclusión}

La capacidad diagnóstica y predictiva de la metodología de evaluación de la dinámica cardiaca basada en la probabilidad y proporciones de la entropía aplicada en casos normales y con arritmias provenientes de la UCl, cuantifico de manera exitosa el nivel de agudización de las dinámicas cardiacas arrítmicas, demostrando la correlación de las predicciones físico-matemáticas de agudización con el diagnóstico clínico convencional.

\section{Dedicatoria}

A nuestros hijos.

A los Maestros Paramahamsa Hariharananda, Paramahamsa Prajnanananda y Bhadrayu Pandya.

\section{Agradecimientos}

Producto derivado del proyecto MED-1345, financiado por la Vicerrectoría de Investigaciones de la Universidad Militar Nueva Granada-Vigencia 2014, y de la Línea de Profundización, Internado Especial y Semillero "Teorías Físicas y Matemáticas Aplicadas a la Medicina".

Agradecemos a la Universidad Militar Nueva Granada, por su apoyo a nuestras investigaciones. Especialmente a la Vicerrectoría de Investigaciones y la Facultad de Medicina. Extendemos un agradecimiento especial a la Dra. Yanneth Méndez, Vicerrectora académica; a la Dra. Nydia Alexandra Rojas, Directora de Investigaciones de la Facultad de Medicina, al Dr. Jorge Luque, Decano de la Facultad de Medicina.

Al Hospital Universitario MEDERI, en especial al Dr. Rafael Riveros por su apoyo al Grupo Insight.

De igual forma al Centro de Investigaciones de la Clínica del Country por su apoyo a nuestras investigaciones; a los Doctores Tito Tulio Roa, Director de Educación Médica, Dr. Jorge Ospina, Director Médico, Dr. Alfonso Correa, Director del Centro de Investigaciones, y a las Doctoras Adriana Lizbeth, epidemióloga y Silvia Ortiz, Enfermera Jefe del Centro de Investigaciones. También a Sandra Rodríguez, enfermera.

\section{Bibliografía}


1. Peitgen H, Strange attractors, the locus of chaos. En: Chaos and Fractals: New Frontiers of Science. Springer-Verlag. N.Y; 1992. p. 655-768.

2. Peitgen H, Jurgens $H$, Saupe D. Chaos and fractals; new frontiers of science. New York: Springer; 1992.

3. Devaney R. A first course in chaotic dynamical systems theory and experiments. Reading Mass: Addison- Wesley; 1992.

4. Girón González Torre, FJ. Determinismo, caos, azar e incertidumbre. [citado 09 Dic 2010]. Disponible en: www.rac.es/ficheros/doc/00327.pdf

5. Sánchez N, Garduño MR, Ritter W, Guzmán SA. Los límites del pronóstico newtoniano y la búsqueda del orden en el caos. Ingeniería Investigación y Tecnología. 2008; 9:171-181.

6. Calabrese JL. Ampliando las fronteras del reduccionismo. Deducción y sistemas no lineales. Revista Psicoanálisis. 1999; 2 : 431-453.

7. Rodríguez J, Narváez R, Prieto S, Correa C, Bernal P, Aguirre G, et al. The mathematical law of chaotic dynamics applied to cardiac arrhythmias. J. Med. Med. Sci. 2013; 4: 291-300.

8. Laplace P. Ensayo filósofico sobre las probabilidades. Barcelona: Altaya 1995

9. Matvéev A. Física molecular. Primera edición, Moscú: MIR, 1987.

10. Tolman R. Principles of statistical mechanics. Primera edición New York: Dover Publications. 1979.

11. Feynman RP, Leighton RB, Sands M. Leyes de la Termodinámica. En: Feynman RP, Leighton RB, Sands M. Física. Vol. 1. Wilmington: Addison-Wesley Iberoamericana, S. A. 1964; p. 44-1, 44-19

12. Pincus SM, Gladstone IM, Ehrenkranz RA. A regularity statistic for medical data analysis. J ClinMonit. 1991; 7:335-45.

13. Guillen $P$, Vallverdú $M$, Rojas $R$, Jugo D, Carrasco H, Caminal P. Dinámica no lineal para caracterizar la variabilidad del ritmo cardiaco en pacientes chagásticos. Sociedad Cubana de Bioingeniería. 2001. Memorias II congreso Latinoamericano de ingeniería Biomédica, Habana 2001, mayo 23 al 25 2001. La Habana Cuba.

14. Vikman S, Mäkikallio TH, Yli-Mäyry S, Pikkujämsä S, Koivisto AM, Reinikainen P, et al. Altered complexity and correlation properties of RR interval dynamics before the spontaneous onset of paroxysmal atrial fibrillation. Circulation 1999; 100:2079_ 84.

15. Rodríguez J. Entropía proporcional de los sistemas dinámicos cardiacos. Predicciones físicas y matemáticas de la dinámica cardiaca de aplicación clínica. Rev Col Cardiol. 2010;17:115-129.

16. Rodríguez J, Prieto S, Domínguez D, Melo M, Mendoza F, Correa C, et al. Mathematical-physical prediction of cardiac dynamics using the proportional entropy of dynamic systems. J. Med. Med. Sci. 2013; 4: 370-381.

17. Rodríguez J, Prieto S, Melo M, Domínguez D, Correa C, Soracipa Y, et al. Entropía proporcional de la dinámica cardiaca aplicada al diagnóstico de pacientes de la Unidad de Cuidados Intensivos. Medicina (Bogotá) 2013; 35: 17-28.

18. Gaziano T, Gaziano M. Global Burden of Cardiovascular Disease. En Braunwald's Heart Disease - A Textbook of Cardiovascular Medicine, 9th ed. Saunders; 2011.

19. Charria D, Sánchez C. Arritmias y trastornos de conducción. Oficina de recursos Educaconales- FEPAFEM. [citado 10 ene 2013] Disponible en : http://www.aibarra.org/Guias/3-2.htm

20. Pineda M, Matiz H, Rozo R. Enfermedad coronaria. Editorial Kimpres Ltda., Bogotá; 2002.

21. Rodríguez J, Álvarez L, Tapia D, López F, Cardona M, Mora J, et al. Evaluación de la dinámica cardiaca de pacientes con arritmia con base en la Teoría de la Probabilidad. Medicina (Bogotá). 2012; 34(1): 7-16.

22. Goldberger A, Amaral L, Hausdorff JM, Ivanov P, PengCh, Stanley HE, Fractal dynamics in physiology: alterations with disease and aging. PNAS 2002; 99: 2466 - 2472.

23. Voss A, Schulz S, Schroeder R, Baumert M, Caminal $P$, Methods derived from nonlinear dynamics for analysing heart rate variability. PhilosTrans A MathPhysEngSci. 2009; 367:277-96.

24. Francesco B, Maria Grazia B, Emanuele G, Valentina $F$, Sara $C$, Chiara $F$, et al, Linear and nonlinear heart rate variability indexes in clinical practice. Comput Math Methods Med. 2012; 2012:219080.

25. Juha S, PerkioMä Ki, Timo H. MäKikallio, Huikuri H. Fractal and complexity measures of heart rate variability. ClinExpHypertens 2005; 2 y 3: 149-58.

26. Huikuri HV, Mäkikallio T, Peng CK, Goldberger A, Hintze U, Moller M. Fractal correlation properties of $R-R$ interval dynamics and mortality in patients with depressed left ventricular function after and acute myocardial infarction. Circulation 2000; 101: 47 -53 .

27. Goldberger A, Amaral L, Hausdorff JM, Ivanov P, Peng Ch, Stanley HE. Fractal dynamics in physiology: alterations with disease and aging. PNAS 2002; 99: 2466 - 2472.

28. Feynman R. Comportamiento cuántico. En: Feynman RP, Leighton RB, Sands M. Física. Wilmington: Addison-Wesley Iberoamericana, S. A. 1964. Vol. 1, cap.37.

29. Rodríguez J. Teoría de unión al HLA clase II teorías de Probabilidad Combinatoria y Entropía aplicadas a secuencias peptídicas. Inmunología 2008; 27(4): 151-66

30. Rodríguez J. Método para la predicción de la dinámica temporal de la malaria en los municipios de Colombia. Rev Panam Salud Pública 2010; 27(3):211-8.

31. Rodríguez J. Nuevo diagnóstico físico y matemático de la monitoria fetal: predicción de aplicación clínica. Momento Revista de Física. 2012; 44: 49-65.

32. Prieto S, Rodríguez J, Correa C, Soracipa Y. Diagnosis of cervical cells based on fractal and Euclidian geometrical measurements: Intrinsic Geometric Cellular Organization. BMC Medical Physics 2014, 14(2):1-9.

33. Rodríguez J, Prieto S, Correa C, Bernal P, Puerta G, Vitery S, et al. Theoretical generalization of normal and sick coronary arteries with fractal dimensions and the arterial intrinsic mathematical harmony. BMC Medical Physics 2010; 10:1-6.

34. Rodríguez J, Prieto S, Correa C, Pérez C, Mora J, Bravo J, et al. Predictions of CD4 lymphocytes' count in HIV patients from complete blood count. BMC Medical Physics. 2013; 13:3.

35. Rodríguez J. Dynamical systems applied to dynamic variables of patients from the Intensive Care Unit (ICU). Physical and mathematical Mortality predictions on ICU. J.Med.Med. Sci. 2015; 6(8): 102-108. 